\title{
Light scattering near and from interfaces using evanescent wave and ellipsometric light scattering
}

\author{
Reinhard Sigel \\ Adolphe Merkle Institute, University Fribourg, Switzerland
}

\begin{abstract}
The broad range of interface light scattering investigations in recent years shows the power and the versatility of these techniques to address new and open questions in colloid and interface science and the soft condensed matter field. Structural information for polymers, liquid crystals, or colloids close to planar or spherical colloidal interfaces are either captured with long range light scattering resolution, or in a complementary approach by high resolution ellipsometric techniques. Of special interest is the dynamic behavior close to or in interfaces, since it determines material properties and responses to external fields. Due to the broad dynamical range and the high scattering contrast for visible light, interface light scattering is a key to elucidate soft matter interfacial dynamics. This contribution reviews experimental and related theoretical approaches for interface light scattering and further gives an overview of achievements based on such techniques.
\end{abstract}

\section{Introduction}

For the quantitative characterization of colloidal dispersions or emulsions and soft matter in general, light scattering is a well established, versatile, and powerful tool [1-4]. The typical size range of the colloids - according to the IUPAC definition 1-1000 nm - fits well to the light wavelength. As a consequence, structural features of a colloid sample significantly affect the scattering properties for visible light. This mapping is not only the basis for a structure characterization by static light scattering (SLS), it additionally determines the optical appearance of dispersions or emulsions. Since colloidal materials are widely applied in food and cosmetic products where esthetic issues are of paramount importance, the optical appearance of colloids and therefore the light scattering properties are of high interest by themselves. Of further relevance are the local mobility and the local relaxation dynamics of dispersions and soft matter like polymers, since these dynamic quantities determine the rheological properties. Dynamic light scattering (DLS) is a convenient tool for the detection of local fluctuations and - via the Stokes-Einstein relation - allows

E-mail address: reinhard.sigel@unifr.ch. the determination of the hydrodynamic radius $R_{\mathrm{h}}$ of particles. Light scattering as a probe of local anisotropy gives access to structure and dynamics in anisotropic soft matter like liquid crystals. Recent experimental developments for the investigation of turbid samples by light scattering are reviewed by Scheffold and Cerbino [5].

Beside bulk properties, also the interface behavior of soft matter is of high interest and relevance. Structure and dynamics of soft matter at the interface determine the mechanical coupling of two adjacent phases. A prominent example of high relevance for applications is a liquid crystal, where the configuration in absence of an external field is settled exclusively by the boundary conditions, i.e. the interfacial coupling. Nano-structured materials like wood, bone, teeth, or artificial material have a huge area of internal interface. As a consequence, the interfacial properties have a significant impact on the behavior of these materials.

The purpose of this contribution is a review of some of the most important applications of light scattering for an investigation of structure and dynamics of soft matter at interfaces. The advantageous properties of light scattering, i.e. the large scattering contrast and a wide experimental window in the time domain which are the footing for bulk light scattering turn out to be fruitful for interface investigations as well. Interface sensitivity of optical techniques can 
be achieved through the usage of an evanescent wave (EW). A keyword search by ISI Web of Knowledge for "evanescent AND scattering" demonstrates the growing interest in such measurements: while during the 1980s the number of annual publications is 3 at maximum, there is a jump to 20 in the early 1990s followed by a steady increase up to 81 in 2007. Clearly, an exhaustive overview on this wide field is out of the scope of this contribution and a focusing is unavoidable. Intentionally excluded are contributions based on total internal reflection microscopy (TIRM), which was introduced by Prieve and Frej in 1990 [6]. This microscopy technique yields the interaction potential between a colloid and a wall and is now widely used. A review on the early TIRM work was compiled by Bike [7]. Also excluded are techniques based on whispering gallery mode sensors [8], where the resonance wavelength of the evanescent waves forming the whispering gallery mode depends with very high sensitivity on refractive index changes in the surrounding. Recent exciting developments of this powerful technique are a label-free single molecule detection due to a thermo-optical effect [9] and the detection of particle Brownian motion close to the detector [10].

The focus in the first part of this work lies on generic scattering measurements with EW illumination, where a variation of the angles of illumination and detection is used to access experimental data in the reciprocal space. So far, a summarizing review on this field is missing. As a foundation for an appreciation of recent developments, also older contributions are discussed for a better overview. Of special interest for interface investigations is the combination of light scattering with an ellipsometric detection scheme, since this catenation enhances the resolution of scattering. Applications based on this approach are discussed as a second part. Excluded here are surface quasi elastic light scattering (SQELS) experiments performed at small angles for the detection of capillary waves at liquid interfaces. For this field, a monograph [11] and reviews by Earnshaw [12] and Cicuta and Hopkinson [13] provide an overview.

\section{Evanescent wave light scattering}

For an implementation of interface sensitive light scattering, Lan et al. used an evanescent wave (EW) created in a total internal reflection geometry as the illumination for a scattering experiment [14*0]. Only a thin volume close to the interface is illuminated and dynamic light scattering reveals the sample dynamics close to an interface. The name evanescent wave dynamic light scattering and its abbreviation EWDLS for this technique were introduced by Marcus et al. [15]. Measurements where only the scattering intensity is of interest will be addressed here as evanescent wave static light scattering (EWSLS), while evanescent wave light scattering (EWLS) denotes light scattering with EW illumination in general. After a brief introduction of the technique, EWLS investigations on colloids, polymers, brushes, and liquid crystals at solid walls as well as EWLS measurements at liquid interfaces are reviewed.

\subsection{EWDLS experiment}

An outline of an EWDLS geometry is sketched in Fig. 1. The interface of two media 1 and 2 with refractive index values $n_{1}$ and $n_{2}$, respectively, is illuminated through the high refractive medium $1\left(n_{1}>n_{2}\right)$. The incident wave vector component $k_{\perp}$ perpendicular to the interface in medium 2 is calculated from Snell's law $n_{1} \sin \varphi=n_{2} \sin \varphi^{\prime}$. In case the angle of incidence $\varphi$ is larger than the critical angle of total reflection $\varphi_{0}=\operatorname{asin}\left(n_{2} / n_{1}\right)$, the angle $\varphi^{\prime}$ of the refracted beam and thus $k_{\perp}$ become imaginary. As a consequence, the exponential factor $\exp \left(i k_{\perp} z\right)$ which describes the oscillations of the refracted beam for real $k_{\perp}$ in case of $\varphi<\varphi_{0}$ becomes the exponential decay $\exp \left(-\left|k_{\perp}\right| z\right)$ of the EW for $\varphi>\varphi_{0}$ [16]. In the $\varphi$-range $\left(\varphi_{0}, 90^{\circ}\right)$ the penetration depth $\zeta=\left|k_{\perp}\right|^{-1}$ of the electrical field decays from formally $\infty$ to $\lambda /\left(2 \pi\left(n_{1}^{2}-n_{2}^{2}\right)^{1 / 2}\right)$, where $\lambda$ is the light vacuum wavelength. Since the light intensity is the squared

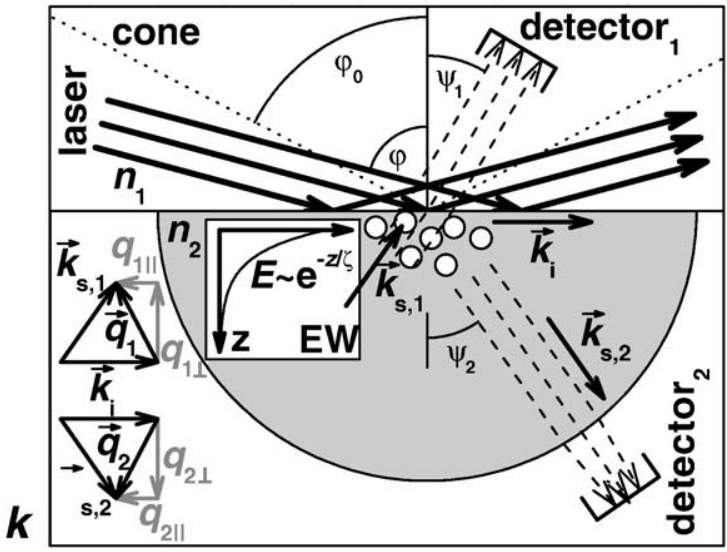

Fig. 1. Sketch of an EWLS scattering geometry. An interface of two media 1 and 2 with refractive index values $n_{1}$ and $n_{2}\left(n_{2}<n_{1}\right)$ is illuminated by a laser beam through the high refractive medium 1 with an angle of incidence $\varphi$ above the critical angle $\varphi_{0}$ of total internal refraction. Scattering from the interfacial region in medium 2 illuminated by the exponentially decaying EW can be observed with an angle $\psi$ either through medium 1 or medium 2 (indicated by an index 1 or 2 ). The scattering vector $\vec{q}$ is the difference of the wave vectors $\vec{k}_{s}$ and $\vec{k}_{i}$ for the scattered and the incident light. It can be decomposed into the components $q_{\perp}$ and $q_{\|}$perpendicular and parallel to the interface.

field amplitude, it decays with $\zeta / 2$. As numerical illustration, the values $n_{1}=1.84$ (Schott glass LaSFN9), $n_{2}=1.33$ (water) and $\lambda=633 \mathrm{~nm}$ result in $\zeta / 2 \approx 40 \mathrm{~nm}$ as smallest intensity penetration depth. This value is large compared to the molecular dimensions, however, it fits well to the colloidal length scale. EWDLS is thus well suited to study interfacial phenomena of colloids and soft matter located in the low refractive medium 2 . At the critical angle where $\zeta$ formally diverges, the beam width and the beam divergence have to be taken into account for the calculation of the illumination profile [17"]. The Poynting vector $\vec{S}=\vec{E} \times \vec{H}$ of an EW indicates an energy flow parallel to the interface and a superimposed oscillation of the field energy perpendicular to the interface. It is often argued that an EW would not be a transverse wave, since the local electric field $\vec{E}$ or magnetic field $\vec{H}$ are not perpendicular to the time averaged energy flow $\langle\vec{S}\rangle[16]$. The argument, however, overlooks the oscillating contribution in $\vec{S}$ which cancels out in the averaging step. When the local energy flow $\vec{S}$ is addressed correctly, an EW is a transverse wave in any space point. Of further interest is the Goos-Hänchen effect of an EW, a shift of the reflected beam along the interface by a fraction of the wavelength (see e.g. [18]).

The scattering of the EW can be observed either through medium 1 or through medium 2. The scattering signal measured through medium 2 is a superposition of a contribution which reaches the detector directly and a second contribution which is originally scattered towards the interface and then reflected to the detector. The detection sensitivity for a particle close to the interface is the product of the exponentially decaying illumination profile of the EW and the interference term of the two contributions in the detection. The reflection at the interface to the high refractive medium 1 involves a phase shift of $180^{\circ}$ for the reflected contribution. For particles located directly at the interface there is a partial destructive interference and the depth resolution is effectively decreased. For a detection of light scattered in the EW in medium 2 through medium 1 , on the other hand, the refraction at the interface has to be taken into account. A corresponding intensity transmission coefficient modifies the detection efficiency while the depth resolution is determined directly by the illumination profile of the EW.

In bulk scattering experiments on isotropic material usually only the magnitude $q$ of the scattering vector $\vec{q}$ is of interest, while the direction of $\vec{q}$ has no meaning. At the interface, in contrast, the wave vector components $q_{\|}$parallel and $q_{\perp}$ perpendicular to the interface have a fundamentally different meaning. The interface introduces 
anisotropy in structure and fluctuations. In addition, the finite extension of the illuminated area perpendicular to the interface results in a smearing in $q_{\perp}$ direction. Since $q_{\perp}$ and $|\zeta|^{-1}$ are both determined by $\lambda$ and are thus of similar magnitude, the smearing is quite substantial. In the experiment, $q_{\|}$results as difference of the parallel wave vector components of incident and scattered light, while $q_{\perp}$ depends only on the detection direction. With $\zeta, q_{\|}$, and $q_{\perp}$ there are three experimental parameters of interest. With an apparatus where only the angles of incidence and detection can be moved within a plane, only a two-dimensional manifold in the $\left(\zeta, q_{\|}, q_{\perp}\right)$-space can be accessed. Preferable is a three-dimensional scattering instrument, as e.g. built by Peter Lang [19]. In such an instrument, the three experimental parameters can be varied independently. For a scattering detection through medium 1 , a special distinction is required. Any light which travels in medium 2 as a plane wave is refracted at the interface into a cone of opening angle $\varphi_{0}$. Light detected in medium 1 at an angle outside this cone cannot be attributed to plane waves in medium 2 . Rather, the scattered field in medium 2 has to be an interface mode, similar to the EW of the illumination. Thus, light detection outside the cone is doubleevanescent [17]. It is connected to $q_{\perp}=0$. Experimentally, Potenza et al. demonstrated that multiple scattered light in medium 2 exits to medium 1 only inside the cone [20].

The presence of an interfacial structure like a polymer brush or a wetting layer affects the refractive index profile across the interface. The deviation from a step profile in turn affects the illuminating profile of the EW, which no longer is a simple exponential. Typically, the refractive index profile $n(z)$ decays monotonously and steadily to $n_{2}$, apart from a first step from the high refractive index $n_{1}$ to the limiting value $n(z \rightarrow 0)$ in the low refractive index medium. The complications by such a refractive index profile are often implicitly neglected, and experiments are described by a nominal EW penetration depth calculated for a step-like transition from $n_{1}$ to $n_{2}$. The presence of a steady refractive index profile, however, allows an additional experimental approach $\left[17^{*}, 21^{\circ}, 22\right]$, which so far hardly has been used. For $\varphi \in\left(\varphi_{0}, a \sin \left(n(z \rightarrow 0) / n_{1}\right)\right)$, the inversion of Snell's law yields a refractive index value $n(\varphi)=n_{1} \sin \varphi$ which is realized within the refractive index profile at a suitable depth $z(\varphi)$. It is this depth where the local light propagation in the direction $\varphi^{\prime}(z)=a \sin \left(n_{1} / n(z) *\right.$ $\sin (\varphi))$ becomes parallel to the interface (i.e. $\varphi^{\prime}(z(\varphi))=90^{\circ}$ ) and the transition from a real $k_{\perp}(z)$ for $z<z(\varphi)$ (propagating wave in $z$ direction) to an imaginary $k_{\perp}(z)$ for $z>z(\varphi)$ (EW in $z$ direction) occurs. In other words, the preset value $\varphi$ is the critical angle at the depth $z(\varphi)$ in the refractive index profile. Local refractive index fluctuations at the depth $z(\varphi)$ switch the local character of $k_{\perp}(z)$ (propagating wave or EW). As a consequence, there is an enhanced scattering contrast for fluctuations in the depth $z(\varphi)\left[17^{\circ}\right]$. The profile of scattering intensity in a scan of $\varphi$ for fixed $\psi$ maps the fluctuation structure within the layer, demonstrated by Sigel and Strobl for the profile of a liquid crystalline wetting layer $\left[21^{\circ}, 22\right]$.

Although EWDLS was introduced already more than 20 years ago and has a lot of interesting applications, the number of publications based on this technique is moderately small. TIRM, in contrast, is by far more used. The reason for the comparably few activities in EWDLS lies most probably in the demanding requirements of the technique and the experimental pitfalls in case of simple implementations. The author heard of three labs where EWDLS was started but finally abandoned because of technical difficulties. Further, no commercial ready-to-use apparatus for EWDLS is on the market, so scientific contributions are bound to home-built experiments. Competing techniques like X-ray or neutron reflectivity are usually situated at large scale facilities, where it is the job of a beamline scientist to take care of technical difficulties and the state of an instrument. Supervised users apply these technique without the need of deeper insight, so a high output of results can be achieved. For an EWLS experiment, in contrast, an experimentalist so far needs the know-how and working effort comparable to a beamline scientist. For a solid liquid interface where medium 1 is typically a hemispheric glass lens, the technical difficulties arise because of the glass part unavoidably contained in the scattering volume. The sample side of the interface in the scattering volume is limited by the evanescent illumination profile. It fills only a small part of the scattering volume and as a consequence the experimental signal is low. Although the scattering of glass is low, the larger filling factor for the glass part of the scattering volume leads to an elastically scattered contribution. The mixing of the signal from the sample with elastically scattered light leads to (at least partial) heterodyne detection, i.e. the superposition of the light scattered by fluctuations in the sample with laser light un-shifted in frequency [1]. In this case, a generalized Siegert relation

$g_{2}(t)=1+2 C_{1} \operatorname{Re}\left(g_{1}(t)\right)+C_{2}^{2}\left|g_{1}(t)\right|^{2}$

connects the intensity autocorrelation function $g_{2}(t)$ measured in the experiment to the field correlation function $g_{1}(t)$ used in data evaluation [19]. The constants $C_{2}=1-\sqrt{1-A}$ and $C_{1}=C_{2}-C_{2}^{2}$ are calculated from the intercept $A$ of $g_{2}(t)$. Via the heterodyne term $\operatorname{Re}\left(g_{1}(t)\right)$, a homogeneous flow of scattering colloids driven e.g. by the light pressure of the incident laser beam (see Section 2.5) can lead to a slow relaxation mode. Of essential importance is the quality of the lens used for an experiment. In the grinding process the surfaces roughness of the lens is reduced to the specified accuracy (a roughness of $\lambda / 20$ is already high quality for grinded surfaces). The remaining interface roughness at smaller scale leads to additional elastically scattered light, sometimes even visible as enhanced intensity at the footprint of the laser at the interface (called "spot" in lab jargon). Significantly smoother glass interfaces are obtained for float-glass, so a cover glass connected by index matching fluid to a grinded lens significantly improves the interface quality. For enhanced intensity, Plum et al. recently used a surface plasmon resonance geometry in order to improve EWDLS sensitivity and data quality [23]. The possibility to measure the diffusion of small, weakly scattering PS colloids $\left(R_{\mathrm{h}}=20 \mathrm{~nm}\right)$ was demonstrated. The enhanced scattering intensity in a surface plasmon geometry compared to EW scattering was demonstrated before by Rothenhäusler and Knoll [24].

The technical requirements are lower for EWDLS at a liquid-liquid interface. Due to interface tension the interface is perfectly flat (apart from capillary waves, which are only visible in small angle scattering, i.e. for a detector position quite close to the reflected beam [11]). Medium 1 has now internal dynamics, so there are no static laser speckles. Still, good vibration isolation is required in order to avoid mechanical excitation of surface waves.

\subsection{Particle diffusion close to a solid interface: dilute regime}

The first work where EWDLS was applied as an interface sensitive dynamic experiment introduced already many essential concepts and properties of the technique [14*0]. Lan et al. [14*] studied the diffusion of colloids with a diameter $2 R=90 \mathrm{~nm}$ close to a solid wall in dilute conditions. The shape of the interface $g_{1}(t)$ determined under heterodyne condition is non-exponential and deviates from the bulk $g_{1}(t)$. Two reasons for this deviation are discussed theoretically. The mirror effect describes the restriction of the colloidal diffusion by the interface, which acts as a solid wall. For the assumed condition with no colloid adsorption at the interface, any diffusion path which hits the interface is reflected. Further, due to the small $\zeta$ which defines the scattering volume perpendicular to the interface, particle number fluctuations become relevant for a suitable choice of $q_{\|}$and $q_{\perp}$. As a consequence, there is a long time tail of $g_{1}(t)$ with a $t^{-1 / 2}$ behavior, which can be rationalized as the diffusive motion within the exponential illumination profile of the EW. A third reason for the non-exponential $g_{1}(t)$ is the wall drag effect, which leads to a decreased diffusion constant for particles close to the interface. The 
wall drag effect is addressed only briefly by Lan et al., but becomes prominent in the induced research activities.

As an extension, Garnier and Ostrowsky studied the diffusion of negatively charged colloids of the same size as above close to a negatively charged interface for different salt concentrations $[25,26]$ ([26] is an extension of the text of [25]). Experimental data as well as corresponding Brownian dynamic simulations based on hydrodynamic wall-particle interactions and static wall-particle interactions described by the DLVO theory (see e.g. [27]) were both fit with the expression derived in [14*], where formally no wall drag effect is incorporated. The results of the fits are interpreted as average interface diffusion constants, where good agreement between theory and experiment is found. A theoretical calculation of the short time diffusion constant serves as a further reference for the data. The average interface diffusion constant is found lower than the bulk value, confirming the wall drag effect. The extent of hydrodynamic slowing down is higher at high salt concentrations, where the particles approach the wall more closely.

By doping lipid vesicles with lipid like charged molecules, Gaigalas et al. obtained negatively and positively charged vesicles $(2 R=42 \ldots$ $44 \mathrm{~nm}$ ) and investigated them by EWDLS [28]. For both samples a slow down of the diffusion close to an interface due to the wall drag effect is observed. The results compare well with theoretical estimations for a homogeneous concentration profile for the negative vesicles and for interface adsorbed locations for the positively charged species. An additional slower mode in $g_{2}(t)$ for the positive vesicles was interpreted as the adsorption process into immobile bound interface states.

By measurements on colloids of sizes $2 R / \mathrm{nm} \in\{97,813,2970$, 11900 , Hosoda et al. checked-out different limits in EWDLS signals for the detection of anisotropic diffusion close to an interface [29]. They introduced the EWDLS description by a complex scattering vector including the exponential decay of the EW as imaginary part, where the finite size of the scattering volume leads to a Lorentzian smearing in $q_{\perp}$ space. In the applied horizontal geometry, the large colloids are affected by gravity and only diffusion parallel to the interface takes place.

Matsuoka and coworkers investigated charge stabilized colloids $2 R=111 \mathrm{~nm}$ for different salt concentrations by EWDLS [30,31] ([31] is an invited feature article which summarizes the content of [30] and further work based on TIRM). The detector angle $\psi=90^{\circ}$ was fixed and no distinction of diffusion parallel and perpendicular to the interface was made. With the intention to distinguish the scattering originating from different depths in the EW, Matsuoka et al. subtracted $g_{1}(t)$ data for two different $\zeta$. The procedure is motivated by a calculation of the difference of electric field strengths for two EW of different penetration depth. This field difference plotted against the distance $z$ to the interface shows a broad peak. It is argued that the difference reflects mainly the scattering signal of the peak position, and a similar depth resolution is assigned to the difference of $g_{1}(t)$ data. The authors claim to observe an "electrostatic effect" on particle diffusion which reaches up $z=800 \mathrm{~nm}$ away from the interface, while they describe a hydrodynamic effect up to $z=400 \mathrm{~nm}$. Opposite to the results of Ostrowsky et al. [25] (see above) and Feitosa and Mesquita [32] (see Section 2.6), the subtraction procedure leads to an apparently faster diffusion for high salt concentration.

Holmqvist et al. built the first EWDLS experiment which allows an independent variation of $q_{\perp}, q_{\|}$, and $\zeta$ based on a 3D triple axis goniometer [19]. They studied the diffusion of charge stabilized colloids $(R=85 \mathrm{~nm}$ ) which behave as hard spheres due to a short Debye length of $3 \mathrm{~nm}$ in a $0.01 \mathrm{M}$ salt solution. A theoretical expression for the initial slope of $g_{1}(t)$ is developed. The instrumentation and the theory enable them to distinguish the wall drag effects for the averaged diffusion constants parallel and perpendicular to the wall. Good agreement with theory is found. The work is extended in reference [33*], which includes a more detailed description of the 3D goniometer, the full derivation of the theoretical expression, an improved data evaluation, and additional measurements on colloids of radius $R=27 \mathrm{~nm}$. Since the scattering intensity of such small colloids is low, they conclude that this size is a lower limit for EWDLS.

A further study of Holmqvist et al. concerns the diffusion of colloids $(R=85 \mathrm{~nm})$ in a mixture with rod-like particles [34]. The rods are fd-virus with a monodisperse length of $880 \mathrm{~nm}$, a cross section diameter of $6 \mathrm{~nm}$ and high stiffness (persistence length $2200 \mathrm{~nm}$ ). They hardly contribute to the scattering signal, but provide an attractive depletion interaction between the colloids and the wall. The interaction range is comparable to $\zeta$. Such a potential increases the particle concentration close to the interface where the wall drag effect is strongest, and therefore it affects the averaged diffusion constants. The potential was investigated by TIRM, where for twice the overlap concentration $c^{*}$ of the rods a strength of $0.5 k_{B} T$ was detected $\left(k_{B}\right.$ : Boltzmann constant, $T$ : absolute temperature). EWDLS investigations at two rod concentrations below and above $c^{*}$ show an effect on the averaged diffusion constants perpendicular and parallel to the wall. An extension of this work is provided in reference [35], where mainly more extended TIRM measurements for a characterization of the depletion potential are added. A comparison of the data in [34] and [35] with a theory describing the depletion interactions by an averaged potential did not provide a satisfying agreement. The authors conclude that the rods act as a second hydrodynamically active species, which leads to a further slowing down of colloid dynamics close to a wall.

\subsection{EWSLS studies on concentration profiles and particle adsorption to a solid wall}

Combining a jet directed towards a wall (wall jet technique) with EWSLS detection, Albery et al. studied the deposition kinetics of colloidal graphite particles onto an indium tin oxide electrode [36]. A microscope slide was used as a waveguide, where EWs are formed at the boundaries. The intensity of the scattered light indicates the amount of adsorbed particles. The wall jet technique yields a controlled mass transport towards the interface and a linear intensity increase with time was observed in the investigated range of low interface coverage (up to $3 \%$ ). With a theoretical model, the radial deposition profile around the jet footprint allows a distinction of mass transfer controlled adsorption (observed for an interface coated with $\mathrm{N}(\beta$-aminoethyl)-G-aminopropyl-trimethoxysilane) and kinetically controlled deposition (a clean glass surface shows a mixed deposition mechanism). Limits of the method are discussed and a kinetic barrier for the glass surface is estimated. In a subsequent investigation, Albery et al. covered the interface with an indium tin oxide (ITO) electrode to vary the interface potential $U$ [37]. For $U>-550 \mathrm{mV}$, the adsorption of the graphite particles $(R=230 \mathrm{~nm})$ was independent of $U$ and showed a radial deposition profile for mass transport controlled deposition. For $U<-550 \mathrm{mV}$, the adsorption rate was lower, but again independent of $U$. A consistent data interpretation for this case was possible with the assumption that the particles are trapped (still mass transport controlled) in a secondary potential minimum further away from the interface, where the EW amplitude is lower and the particles produce a weaker scattering signal. Particles collected in the secondary minimum could be collapsed to the primary minimum by a variation of $U$. The existence of a secondary minimum was dependent on the type of interface, and a quantitative model for the observation of a secondary minimum is discussed.

Schumacher and van de Ven studied EWSLS of charged colloids ( $R=46.2 \mathrm{~nm}$ ) close to a charged wall [38]. The change in scattering intensity upon a variation of $\zeta$ was compared to predictions based on a thermal particle concentration profile derived from DLVO particlewall interactions, where good agreement was found. From EWDLS measurements, only the variation of the $g_{2}(t)$ intercept was interpreted, the information content of which is equivalent to the 
static intensity. The careful measurement protocol employed changes of the sample concentration without a variation of the scattering angle and the angle of incidence. This laborious procedure was repeated for several angle settings, presumably to avoid the problems due to static laser specles described in 2.1 .

With the wall jet technique and EWDLS, Xia and van de Ven studied the adsorption kinetics of unilamellar or multilamellar lipid vesicles (liposomes, $2 R=90 \ldots 400 \mathrm{~nm}$ ) to a quartz surface [39]. The effects of flow rate, liposome size, $\mathrm{pH}$, and salt concentration on the adhesion rate were investigated. A maximum adhesion rate was observed for intermediate flow rates. $\mathrm{Ca}^{2+}$ ions lead to an enhanced adhesion compared to $\mathrm{Na}^{+}$ions. As a continuation, Polverari and van de Ven measured the adsorption of 3 types of negatively charged colloidal particles $(2 R=145 \ldots 245 \mathrm{~nm}$, PS copolymers with acrylic acid or itachonic acid, or pure PS) to a fused quartz and a ZnS interface $\left[40^{\circ}\right]$. The particles were investigated with and without poly(ethylene oxide) (PEO) polymers $\left(M_{w}=100 \ldots 350 \mathrm{~kg} / \mathrm{mol}\right)$ adsorbed to their interface, where polymer adsorption layers of $2 \ldots 22 \mathrm{~nm}$ were realized. Adsorption was measured as a function of $\mathrm{NaCl}$ concentration, where a maximum adsorption rate was reached at $0.5 \mathrm{M} \mathrm{NaCl}$ with no further increase at higher salt concentration. The bare particles adsorb much faster to the interface, and no adsorption at all was observed for PEO layers above $15 \mathrm{~nm}$ thickness. In a complementary work on the same particles at low salt concentration where interface adsorption is absent, Polverari and van de Ven studied the effects of particle diameter, salt concentration and flow rate on the interfacial concentration profile, examined by EWSLS [41]. The theory for a latex concentration profile in a stagnation point flow towards the interface is reviewed, which is based on a Boltzmann distribution involving static DLVO interactions and hydrodynamic effects. Agreement between the experiments and the theory is concluded.

\subsection{Particles close to a solid interface: elevated concentration}

For elevated particle concentration, only a few investigations by EWDLS were performed so far. Pouligny et al. used EWSLS for an investigation of the interface of a colloidal crystal to a container wall [42]. They developed a theory for the peak locations for an EW illumination in a coplanar geometry and for the peak intensities upon a variation of $\zeta$. The crystal was formed by charge stabilized colloids $(2 R=91 \mathrm{~nm})$ without added salt. Several measurements were recorded and the crystal was shear-melted in between the measurements. The results are compatible with an 110 plane of a bcc crystal parallel to the interface. No surface reconstruction of the crystal was observed, but a smaller lattice parameter close to the interface is reported.

Schmidt et al. studied the film formation of poly-methyl methacrylate/butyl-acrylate colloids $(2 R=93 \mathrm{~nm})$, which was slowed down to $3000 \mathrm{~s}$ [43]. Two relaxation processes were observed in $g_{1}(t)$, which were identified with the $\alpha$ and $\beta$ process in glassy systems. The relaxation time of the slower process slows down during film formation.

Voudouris et al. investigated index matched PMMA colloid ( $R=118 \mathrm{~nm}$, sterically stabilized) polymer (gyration radius $R_{\mathrm{g}}=$ $90 \mathrm{~nm}$ ) mixtures in a tetralin/decalin solvent mixture at a colloid volume fraction $\Phi=0.3$ [44]. Due to depletion interactions, the system phase separates in a polymer rich "gas"-phase $(\Phi=0.0013)$ on top of a colloid rich "liquid"-phase $(\Phi=0.42)$. The scattering is dominated by the colloids. In bulk measurements, the effect of the depletion interaction is visible by an enhanced low angle scattering of the liquid phase compared to measurements on a colloid dispersion of similar concentration without polymers. Such a dispersion of elevated concentration shows similar dynamics in the bulk and in EWDLS interface measurements. For the "liquid"-phase (polymers included) with slower bulk dynamics, in contrast, a further slowing down at the interface is observed and attributed to depletion interactions. By
EWDLS the authors showed, that the "liquid"-phase builds up a wetting layer at the container walls of the top part of the scattering cell, where the bulk is the "gas"-phase. The dynamic signature of the liquid phase within the wetting layer does not depend on $\zeta$ and the authors conclude that this layer is thicker than 1-2 $\mu \mathrm{m}$. Further work on EWLS within wetting layers $\left[21^{\circ}, 22,45\right]$ is addressed in Section 2.8.

A detailed investigation of the concentration dependence up to $\Phi=0.42$ for the near-wall diffusion of colloids ( $R=118 \ldots 183 \mathrm{~nm})$ was performed by Michailidou et al. [46 $]$. Again the solvent was a tetralin/cisdecalin index matching mixture. Measurements for $q$ below the structure factor maximum due to short range liquid order yielded the collective diffusion constant, while high- $q$ measurements above the structure factor maximum was identified with the self diffusion. For $\Phi>0.35$, the interface dynamics superimpose to the bulk dynamics, in contrast to the slowing down of wall dynamics at lower $\Phi$ (see discussion of references [19] and [26] above). The absence of the wall drag effect is discussed as a screening of a long range contribution to hydrodynamic interaction at high $\Phi$. From theoretical side, the initial slope calculation for $g_{1}(t)$ under EW illumination for a test particle in dilute condition by Holmqvist et al. $\left[19,33^{*}\right]$ was generalized to higher volume fraction.

\subsection{Mie scattering and scattering forces for isolated spheres in an evanescent wave}

Theoretical expressions for the calculation of scattering intensities for Mie scattering of spherical particles in an EW were derived by Chew et al. [47] and corrected later by Liu et al. [48]. Total cross sections for extinction and scattering for an EW were introduced and calculated by Quinten et al. [49]. Further theoretical work was stimulated by an experiment of Kawata and Sugiura, who observed the movement of PS spheres ( $2 R=1 \mu \mathrm{m}, 6.8 \mu \mathrm{m}, 27 \mu \mathrm{m})$ and glass particles $(2 R=2 \mu \mathrm{m}, 8 \mu \mathrm{m})$ by the light pressure of an EW [50"]. The movement was faster for $s$-polarized light. A typical speed of $8 \mu \mathrm{m} / \mathrm{s}$ was observed for PS spheres with $2 R=6.8 \mu \mathrm{m}$. Almaas and Brevik calculated the scattering forces of an EW, which are stronger for $p$ polarization [51]. An interpretation of this result opposite to the experimental finding in reference $\left[50^{\circ}\right]$ was attributed to a friction effect. Beside the force parallel to the interface which moves the colloids, there is also a normal force which tends to move the particles into higher field regions, like in optical tweezers. Since $p$-polarization results in a stronger normal force, enhanced friction is expected. The maximum scattering force was found for a value $\alpha=3$ for the size parameter $\alpha=2 \pi R / \lambda$. While in all previous theories the EW was created by a plane wave in the high refractive index medium, Chang et al. calculated the light pressure of an EW created by a Gaussian beam illumination [52]. As an extension, they considered also the effect of multiple scattering which was neglected so far[53]. Multiple scattering occurs when light scattered by the sphere is reflected by the interface and hits the sphere another time. Zvyagin and Goto compared the multipole expansion in Mie theory with group theoretical methods for the calculation of the scattering of an EW [54]. The group theoretical methods were introduced by Pendleton for plane waves and lead to less complicated formulas [55]. Doicu et al. applied the T-matrix method and discrete source method to an EW geometry [57]. These methods yield the scattering properties also for non-spherical particles. Liu et al. calculated morphology-dependent resonances (MDR) of spherical particles close to a substrate, illuminated either by a plan wave or an EW [56]. Ganic et al. performed numerical calculations of MDRs of spheres illuminated by an EW in the full angular range [58]. Experiments on MDRs of spheres (size 1,2, or $6 \mathrm{~nm}$ ) illuminated by an EW were reported by Wu [59]. A significant variation in scattering power of different spheres in TIRM measurements is attributed to MDRs. 


\subsection{Particles confined in a planar geometry}

The dynamics of colloids confined by two plates to a quasi twodimensional geometry has been accessed by several techniques. A recent reference list is included in reference [60]. Here, only light scattering investigations on confined geometries are addressed. The interface sensitivity of EWDLS for such geometries is of minor importance, as the movement of colloids perpendicular to the interface is suppressed and measurements for $\varphi$ above or below the critical angle $\varphi_{0}$ yield the same information.

The investigation of rhodopsin diffusion in multilamellar films of the photoreceptor membrane (PRM) by Selser et al. in 1982 appears to be the first application of EWDLS [61*]. Roughly $10^{3}$ membrane layers of 10-20 $\mu \mathrm{m}$ thickness were deposited on a waveguide, and EWDLS was detected at $90^{\circ}$ scattering angle. The observed slow diffusion $D=2 \ldots 11 \cdot 10^{-15} \mathrm{~m}^{2} / \mathrm{s}$ was attributed to rhodopsin aggregation.

Introducing colloidal particles $(R=1000 \mathrm{~nm})$ into a wedge slit between two prisms, Feitosa and Mesquita investigated different separation distances $h$ of the confining interfaces [32]. Theoretical formulas for the mirror effect, the wall drag effect and double layer repulsion are summarized. DLS measurements (plane wave illumination) in confined geometry with gravity either parallel or normal to the surfaces were complemented by an investigation of particles sedimented to an interface in an unconfined geometry, where the electrostatic interactions between particles and wall are varied by a change of the salt concentration. A linear superposition of the Stokes forces due to the wall drag effects at the two confining interfaces was assumed in a theoretical description. This assumption gives a reasonable agreement for the diffusion perpendicular to the interfaces, however, for the diffusion parallel to the interfaces significant deviations were observed at small $h$. The theoretical formulas for the wall drag effect were used to transform the measured diffusion into the average distance of the sedimented particles to the repulsive wall. A decrease of the distance with increasing salt concentration was found, in accordance with expectation.

A comparison of video microscopy and EWDLS on the diffusion of uncharged poly(methyl-methacrylate) (PMMA) colloids $(2 R=$ $1000 \mathrm{~nm}$ ) confined in a slit (wall spacing: $3 \mu \mathrm{m}$ ) performed by Marcus et al. provided good agreement between the two techniques $\left[15^{\circ}\right]$. A theoretical expectation for a transition of the self diffusion constant in two dimensions linear in $\ln (t) / t$ was confirmed experimentally, although the magnitude of the observed transition was larger by a factor 7 compared to the theoretical expectation. The deviation was attributed to the experimental geometry, which is not a true two-dimensional system. A Gaussian model for single particle motion within the many particle problem provided good agreement with the data. The wall drag effect was not explicitly taken into account in this work.

For a theoretical description of the wall drag effect of colloids confined between two walls, Lobry and Ostrowsky adapted the method of flow field reflection to two walls [62"]. Multiple reflections at the two walls lead to an infinite series of single wall problems, the analytical solution of which is known. Experiments on the diffusion perpendicular to the wall for colloidal particles of two sizes $(R=39 \mathrm{~nm}, 110 \mathrm{~nm})$ showed good agreement with the theoretical calculations. The theory was refined by Benesch et al. [63].

\subsection{Polymers and brushes at a solid interface}

Theoretical investigations by Gao and Rice paved the way for EWLS on polymers close to an interface $[64,65]$. Their first work describes EWSLS by isolated polymer chains adsorbed to an interface [64]. Two geometries with the detector either in the interface plane or in the reflection plane are introduced and general expressions for the scattering of a single chain (denoted as structure factor) are derived. Specific analytical and numerical calculations concern the model of a rod-like polymer anchored to the interface. An extension of this work handles the case of Gaussian chains which are either anchored to the interface or freely moving in the vicinity of the interface [65]. Intermolecular interference for elevated interface coverage is treated by a virial expansion. The effect of polymerinterface interactions on the chain dimension perpendicular to the interface and the corresponding structure factor are discussed. Rosa and Dahler incorporated an orientation distribution function for the rod-like polymers at the interface and reported numerical calculations for the structure factor [66].

EWLS experiments on polymers and brushes were performed by the Heraklion group $\left[67^{\circ}, 68,69^{*}, 70,71,72^{\circ}, 73\right]$. A brush is formed by polymers anchored to an interface when the distance between grafting sides is significantly smaller than the extension of the anchored polymers. As a consequence, the polymers are stretched in the direction perpendicular to the interface. The first systems investigated by Fytas et al. were four different poly(ethylene oxide)-blockpolystyrene (PEO- $b$-PS) diblock copolymers of different molecular weight in toluene [ $\left.67^{\circ}\right]$. While the short PEO block (51...167 monomer units) adsorbs to the interface, the longer PS block $(730 \ldots 4790$ monomer units) remains dissolved and forms the brush of thickness $L_{0}=45 \ldots 130 \mathrm{~nm}$ with a grafting density $\sigma=0.19 \ldots 2 /(10 \mathrm{~nm})^{2}$. Homodyne data evaluation yielded an exponentially decaying $g_{1}(t)$ with a relaxation time in the ms range. The related intensity raised quadratic with $q$, while the relaxation time increases with increasing $q$ (no distinction between $q_{\|}$and $q_{\perp}$ was made). The scattering is attributed to concentration fluctuations in the brush, where the finite possible stretching of polymer chains leads to a suppression of long wavelength (low q) fluctuations.

The measurements of reference [67"] induced two theoretical contributions. With a mean field theory, Likhtman et al. addressed EWSLS of a brush in good solvent conditions [74,75] ([75] is a summary of [74]). Free energy contributions for brush deformation consist of chain stretching, an osmotic contribution, and a gradient term accounting for surface distortions at the brush boundary. The theory considers $q_{\|}$and $q_{\perp}$ separately and produces a weak maximum in the scattering intensity for a variation of $q_{\|}$, keeping $q_{\perp}$ fixed. The stronger maximum observed experimentally in reference [67 ${ }^{\circ}$ is attributed to the special trajectory in $\left(q_{\|}, q_{\perp}\right)$ space when the detector angle is varied in the experiment. The trajectory is modeled in the theory and qualitative agreement is found for three of the brushes examined in reference [67"], while for the thickest brush a deviation is concluded. A theoretical investigation for EWDLS on brush dynamics for a scattering vector parallel to the interface was performed by Semenov and Anastasiadis [76]. Three relaxation modes of a brush were discussed. The relaxation times of a fast cooperative diffusion of polymer segments together with the solvent and a drag motion where polymer and solvent movement are not correlated were predicted for solvent and polymer parameters in reference $\left[67^{\circ}\right]$ as $0.5 \cdot 10^{-6} \mathrm{~s}$ and $1 \ldots 2 \cdot 10^{-5} \mathrm{~s}$, respectively. Since these times are significantly faster than the experimental result in reference [67"], an additional slow anchor sliding mode was introduced. The anchoring of the polymers relies on adsorption of the PEO block [67"] and therefore can be mobile. A two-dimensional formation of islands of PEO blocks at the interface is predicted, similar to the formation of "furry discs" of insoluble block copolymers on liquid interfaces [77] (see Section 2.9). The anchor sliding mode allows a qualitative matching of the theory with the experiment $\left[67^{\circ}\right]$, and it is concluded that the two fast modes are not resolved experimentally.

An investigation of the interface dynamics of the hairy rod polymer poly ( $p$-phenylene (PPPS) in toluene for four number averaged contour lengths $L_{n}=11 \ldots 134 \mathrm{~nm}$ was performed by Loppinet et al. [68]. Adsorption from a semidilute solution led to equilibration within $20 \mathrm{~h}$. By a variation of the angle of incidence around $\varphi_{0}$, distinct differences in $g_{1}(t)$ for bulk DLS $\left(\varphi<\varphi_{0}\right.$, refracted beam in medium 2$)$ and EWDLS sensitive to the interface $\left(\varphi>\varphi_{0}\right.$, EW in medium 2$)$ were 
observed. Rotational dynamics was accessed by depolarized light scattering [1]. While in the bulk the rotational dynamics showed a broad relaxation time distribution with a strong concentration dependence (in a range up to $20 \% \mathrm{wt}$ ), the interface dynamics showed a single exponential shape with a relaxation time in the $\mathrm{ms}$ range with only very weak dependence on concentration, $L_{n}$, and $\zeta$. The interface dynamics was attributed to rotational motion of Kuhn segments(PPPS persistence length $\approx 25 \mathrm{~nm}$ ) in an adsorption layer with an absence of overall chain rotation. Polarized DLS showed concentration fluctuations for bulk measurements, while in EWDLS a similar signal than for depolarized light scattering was observed, i.e. the rotational dynamics dominated also polarized measurements.

First EWDLS measurements on a covalently anchored PS brush (PS: $M_{w}=1600 \mathrm{~kg} / \mathrm{mol}$, polydispersity $M_{w} / M_{n}=2$; dried brush thickness $95 \mathrm{~nm}$ ) with significantly higher grafting density $\sigma=16$ / $(10 \mathrm{~nm})^{2}$ than in reference [67 ] were performed by Yakubov et al. [69*']. Exposing the brush to good solvents (dioxane and toluene), an exponential relaxation with diffusive relaxation rate $\Gamma=D_{c} q^{2}$ was observed, independent of the angle between the scattering vector and the interface normal. $D_{c}$ is connected to the correlation length $\xi=k_{B} T /$ $\left(6 \pi \eta D_{c}\right)$ ( $\eta$ : solvent viscosity). A constant value $\xi=4.6 \mathrm{~nm}$ at $T=20{ }^{\circ} \mathrm{C}$ and $T=40{ }^{\circ} \mathrm{C}$ indicates a temperature independent brush profile for dioxane representing a good solvent. For a variation of the solvent quality, the brush was exposed to cyclohexane. The solvent quality decreases with $T$, and at $T_{\theta}=35{ }^{\circ} \mathrm{C}$ cyclohexane becomes a theta-solvent for linear polymers in bulk, i.e. excluded volume interactions of polymer chains are screened. A two step decay of $g_{1}$ $(t)$ was found in EWDLS. The broad slow decay (described by a stretched exponential $\exp \left(-[t / \tau]^{\beta}\right.$ with $\left.\beta=0.32\right)$ shows a diffusive behavior with $q$ and increases in amplitude for decreasing $T$. The relaxation time $\tau$ followed a scaling relation $\tau_{s} \sim\left(T-T_{C}\right)^{-x}$ with $T_{c}=28{ }^{\circ} \mathrm{C}$ and $x=2.6$. A lateral phase segregation at the lowest $T$ was excluded because it would produce higher scattering power than observed experimentally. The exponential diffusive fast process yields $\xi$, which increases from $\xi=5 \mathrm{~nm}$ at $T=58.4{ }^{\circ} \mathrm{C}$ to $\xi=21 \mathrm{~nm}$ at $T=16{ }^{\circ} \mathrm{C}$. No critical behavior was observed at $T_{\theta}$ in this investigation of the dynamic behavior of a polymer brush. Interestingly, an effect of polymer morphology on the value of $T_{\theta}$ has been reported for the PS/ cyclohexane system, with $T_{a}=28{ }^{\circ} \mathrm{C}$ for ring polymers, similar to the value of $T_{c}$ for the slow process [78]. As an extension of reference [69"], Michailidou et al. varied brush parameters in an EWLS study on three PS brushes $\left(\sigma=1.6 \ldots 16 /(10 \mathrm{~nm})^{2}\right)$ as well as a poly $(n$-butyl acrylate) brush $\left(\sigma=6 /(10 \mathrm{~nm})^{2}\right)$ in good solvent conditions [70]. The collective diffusion detected in the exponential decay of $g_{1}(t)$ is inversely proportional to the solvent viscosity and increases with $\sigma\left(D_{c} \sim \xi^{-1} \sim \sigma^{1 / 2}\right)$. The scattering intensity grows linearly with penetration depth, which implies a homogeneous distribution of concentration fluctuations in the brush. An unexpected strong increase of the scattering intensity with $\sigma$ was observed. The discussion compares the fluctuations in a brush and in a semidilute bulk polymer solution. A study of the same PS brushes in dioxane [71] extended the investigations in the $\theta$ solvent regime of reference [69*0]. As before a two step decay is found for this regime. As for the good solvent case the fast collective diffusion increases with grafting density, while for the slow relaxation rate an opposite trend is observed. Different than in reference [69*], the slow mode relaxation time appears now independent on $q$; the difference is attributed to the considerable width of the relaxation time distribution. While for the two brushes of lower $\sigma$ $\left(1.6 /(10 \mathrm{~nm})^{2}\right.$ and $\left.5 /(10 \mathrm{~nm})^{2}\right)$ the amplitude ratio of fast and slow mode is independent of the EW penetration depth $\zeta$, the slow mode contribution increases with $\zeta$ for the brush with $\sigma=16 /(10 \mathrm{~nm})^{2}$, which hints to a stronger amplitude of the slow mode in the outer part of the brush. The observed relaxation times of the two modes were independent of $\zeta$. In the discussion, the two relaxations are related to the behavior of a semidilute bulk polymer solution under $\theta$ conditions, where also two relaxation modes are observed.
The diffusion behavior of particles close to a wall covered with a polymer brush $\left(\sigma=5 /(10 \mathrm{~nm})^{2}\right.$, estimated thickness: $1 \mu \mathrm{m}, \xi=5 \mathrm{~nm}$; PS: $M_{w}=1000 \mathrm{~kg} / \mathrm{mol}$ ) was investigated by Filippidi et al. [72']. While PMMA colloids $(R=120 \mathrm{~nm})$ represent hard particles, a 64-armed star block copolymer (inner part PB, arms PS, $R_{h}=20 \mathrm{~nm}$ ) was taken as an example for a soft particle. The heterodyne scattering signal was dominated by the particles and the scattering of the brush was neglected. Based on measurements at different $\zeta$ and a simple model with a step-like particle concentration profile, the authors determined the penetration of the particles into the brush. While measurements for a collapsed brush in dodecane are compatible with the dry brush thickness of $30 \mathrm{~nm}$, the colloids and the stars penetrate the polydisperse brush in decalin as a marginal solvent up to depths of $230 \mathrm{~nm}$ and $160 \mathrm{~nm}$, respectively. The depth indicates the distance from the solid wall where the brush is grafted. A non-exponential shape of $g_{1}(t)$ was observed and the short time diffusion constant is extracted from the initial decay. For small $\zeta$, a dynamic slower than in the bulk but faster than for wall drag at solid interfaces is observed for the hard colloids. For the star block copolymers on the other hand, a distinct dependence of the slow down on $\zeta$ was found, which was attributed to friction within the polymer brush. The work was extended by Michailidou et al., who investigated the effect of grafting density on particle penetration and diffusion in a brush [73]. Three PS brushes (PS: $M_{w}=1000 \mathrm{~kg} / \mathrm{mol}$ ) of different grafting density were exposed to PS nanogels of different size $(R=16 \mathrm{~nm}, 42 \mathrm{~nm})$ which were shown to behave as hard spheres. The depths of penetration $L$ were similar for the two nanogels but depend strongly on $\sigma$ ( $L=375 \mathrm{~nm}$ for $\sigma^{-1 / 2}=3 \mathrm{~nm}, L=230 \mathrm{~nm}$ for $\sigma^{-1 / 2}=5 \mathrm{~nm}$, and $L=100 \mathrm{~nm}$ for $\sigma^{-1 / 2}=8 \mathrm{~nm}$ ). It should be noted, however, that the brush and the nanogels both consist of swollen PS and the results for $L$ might thus be affected by scattering contrast issues, since the visibility of the particles changes in the concentration profile of the brush. The slowing down of the nanogel diffusion is stronger in the brush compared to a hard wall interface. The diffusion constants for the two nanogels were almost independent of $\sigma$, but a significant stronger slowing down was observed for the small nanogels. The authors speculate that a specific length scale in the outer part of the brush might be responsible for the unexpected stronger effect on the small nanogels.

\subsection{Liquid crystals and liquids close to a solid interface}

The interface dynamics of a nematic liquid crystal (LC) was studied by Copic, Clark, and coworkers [79-81]. A first theoretical work predicts $g_{1}(t)$ determined by EWDLS for the case that a coupling of a shear velocity and the field of the nematic director $\hat{n}$ can be neglected [79]. As for bulk light scattering, there are a splay-bend fluctuation mode determined by the LC elastic constants $K_{1}$ and $K_{3}$ and a twistbend mode involving $K_{2}$ and $K_{3}$. By a suitable choice of the polarization directions of incident and detected light and a suitable geometry, these modes can be studied separately for planar ( $\hat{n}$ within the interface) as well as for homeotropic ( $\hat{n}$ perpendicular to the interface) interface alignment. The anchoring energy of the LC at the interface is predicted to affect weakly the shape of $g_{1}(t)$. A long time tail of $g_{1}(t)$ proportional to $t^{-3 / 2}$ is predicted which is steeper than the one for diffusing particles (see discussion of reference [14*] in 2 above). An extended theoretical work based on continuum nematodynamics takes also a coupling between director field and shear into account [80]. Since at the interface the fluid velocity vanishes, the fluid can no longer follow the director motion. The viscous drag for a director reorientation at the interface is effectively increased. This effect is found to be particularly strong for the bend mode. As a consequence, a slow surface relaxation mode which decays exponentially into the bulk is predicted. The theory was verified in an experimental work on the LC 5CB [81]. For the relaxation time of a twist-bend surface mode a slow down by a factor 1.66 compared to 
the bulk mode was found. Further, an interface anchoring energy of $7.14 \cdot 10^{-5} \mathrm{~J} / \mathrm{m}^{2}$ was extracted from the data. The value fits well to reference values obtained by other techniques.

An EW illumination combined with Brillouin scattering detection was used by Bonn and Wegdam to study sound propagation modes in a wetting layer of a demixed binary fluid system [45]. The combination of cyclohexane and methanol formed a cyclohexane rich phase on top of a methanol rich phase. The latter phase forms a wetting layer at the interface of a quartz semicylinder at which the EW is generated. Beside bulk sound modes, EW Brillouin scattering in the upper part of the container where the wetting layer neighbors the cyclohexane rich bulk phase yielded two surface modes with a dispersion relation linear in $q_{\|}$. The sound propagation speed and the damping of the slower surface mode resemble the bulk mode in the methanol rich phase, while the other surface mode is faster than the bulk modes in either liquid phase. An interpretation based on hydrodynamic calculations assumed a high frequency visco-elasticity in the wetting layer. An EWDLS investigation on a wetting layer of a mesoscopic colloidal fluid [44] is addressed in Section 2.4.

A different type of wetting is orientational wetting in nematic LCs, where just above the phase transition temperature $T_{n i}$ where the bulk is in the isotropic phase the interface is covered by a thin nematic layer. Sigel and Strobl investigated fluctuations of the nematic wetting layer in the LC CB8 for homeotropic alignment [21]. For a suitable polarization direction of the light and $\varphi$ in the right range, enhanced EWSLS indicated orientation fluctuations within a limited temperature range of $0.3 \mathrm{~K}$ above $T_{n i}$. Within this temperature range EWSLS intensity and EWDLS relaxation time showed a significant dependency on $q_{\|}$, in contrast to the behavior at higher temperatures or the bulk scattering of the isotropic phase. The disappearing of EWSLS intensity at higher temperatures is interpreted tentatively as a prewetting transition of the layer. The work is extended by further EWSLS measurements, a characterization of the wetting layer by elliposmetry, a measurement of the isotropic bulk correlation length $\xi_{\mathrm{LC}}$, and an extended interpretation [22]. Directly at $T_{n i}$, comparable values for the layer thickness $6.9 \mathrm{~nm}$ and $\xi_{\mathrm{LC}}=5.6 \mathrm{~nm}$ were obtained. The scattering intensity recorded in a scan of $\varphi$ gives access to the refractive index profile of the wetting layer (see Section 2.1) and the LC order parameter value $S_{0}$ directly at the interface was determined. A steady behavior of $S_{0}$ at the bulk phase transition $T_{n i}$ was observed. An amplification mechanism for EWSLS in a refractive index profile is discussed (see also [17 $]$ ). The $q_{\|}$dependence of EWSLS and EWDLS hints to a characteristic length scale in the interfacial plane. The steady temperature dependence of ellipsometry data excludes the occurrence of a prewetting transition. The limited temperature range above $T_{n i}$ where enhanced EWSLS is observed is interpreted as stability range of an interface fluctuation mode carried by the nematic wetting layer.

\subsection{Liquid-air and liquid-liquid interfaces}

An EWLS study at the liquid-air interface by Lin et al. considers polystyrene-block-polymethylmethacrylate (PS- $b$-PMMA) diblock copolymers (molecular weights $M_{w}$ : PS $M_{w}=880 \mathrm{~kg} / \mathrm{mol}$, PMMA $M_{w}=290 \mathrm{~kg} / \mathrm{mol}$ ) sprited on top of an aqueous surface $\left[77,82,83^{\circ}\right]$. Experiments were performed on a Langmuir balance with temperature controlled housing, a multi-angle fiber optical detector for EWSLS and a single mode fiber for EWDLS. EWSLS showed the presence of nearly monodisperse disc-like aggregates on the interface and the absence of isolated polymers [82]. While there is no scattering contrast from the PMMA, the dense PS core has a diameter of $380 \mathrm{~nm}$ with a weak concentration dependence. The block copolymer layer is patchy for an interface coverage below 1 monomer per $0.18 \mathrm{~nm}^{2}$ and becomes homogeneous above. A peak in EWSLS at high interface coverage could be fitted with a two-dimensional hard disc model. In an extension with improved interpretation the 2D aggregates are denoted as "furry discs", in reference to the brush-like PMMA boundary around the PS core [77]. The aggregation number was estimated as 240 polymers per "furry disc." The authors demonstrated, that the interface homogeneity and therefore the data quality in EWSLS and EWDLS data got significantly improved by either two surface compressions in the Langmuir trough or by spriting the pure solvent chloroform on the polymer covered interface (it was shown later that the spriting of chloroform slightly reduces the interface pressure [ $\left.\left.8^{\circ}\right]\right)$. EWDLS yielded a two step decay in $g_{1}(t)$. The fast diffusive process was attributed to isolated "furry discs," while the slow decay was assigned to islands of discs attached together. Estimations based on EWSLS and EWDLS gave a lower limit of $20 \mu \mathrm{m}$ for the radius of the islands. The in-plane diffusion constant of the fast process was transformed to a two-dimensional hydrodynamic radius of $410 \mathrm{~nm}$. The significantly larger value than the PS core size was attributed to the extension of PMMA chains stretched on the interface around the core. This picture was further supported by surface pressure measurements. In a later work, the authors used the same system to cross check theoretically predicted logarithmic divergences for the diffusion coefficient in a two-dimensional system [83"]. At elevated interface coverage where EWSLS shows a peak at $q_{m}$, EWDLS detects collective diffusion for $q_{\|}<q_{m}$ and self diffusion for $q_{\| \prime}<q_{m}$. In both regimes, agreement with theory was found.

Stocco et al. investigated the dynamics of capillary waves at a water-air interface $\left[17^{\circ}\right]$. The usage of an EW geometry with illumination from the water side gives access to $q_{\|}=0.2 \ldots 0.9 \mu \mathrm{m}^{-1}$. These $q_{\|}$-values are larger than in surface quasi elastic light scattering (SQELS) [11] and even significantly exceed the range investigated by X-ray photon correlation spectroscopy (XPCS) so far. The obtained results for the interface tension in the $q_{\|}$range are larger than data obtained from macroscopic, static methods. Possible experimental causes for this observation are discussed and excluded. A tentative interpretation assigns the enhanced interface tension to charge effects at the aqueous interface. Further, a narrow scattering peak was observed for EW illumination ( $\varphi>\varphi_{0}$, fixed) when the detection angle $\psi$ was scanned. The location $\psi=\varphi_{0}$ of the peak resembles the Yoneda peak, however, the detected scattering peak is significantly higher and narrower than a Yoneda peak. The peak was weakened by the adsorption of a symmetric amphiphilic polyisoprene-block-poly (ethylene oxide) (PI- $b$-PEO) block copolymer $\left(M_{w}=16.4 \mathrm{~kg} / \mathrm{mol}\right)$ to the aqueous interface. The discussion relates the peak to a second order scattering effect with a scattering contrast mechanism only operational at the critical angle $\varphi_{0}$. The mechanism is affected by the refractive index profile of the interface, and was related to the observation of an enhanced EWSLS scattering intensity for a LC wetting layer $\left[21^{\circ}, 22\right]$ (see Section 2.8). A further work of Stocco et al. describes the diffusion of colloidal particles at an alkane-water interface [84]. An apparatus is introduced which allows an independent variation of $q_{\|}, q_{\perp}$, and $\zeta$ in a three-dimensional EW scattering geometry especially suited for liquid-liquid and liquid-air interfaces. EWDLS was independent on $q_{\perp}$, proving that the particles are trapped by the liquid-liquid interface via the Pieranski potential [85]. The twodimensional in-plane diffusion is significantly slowed down compared to the bulk diffusion.

\section{Combination of light scattering and ellipsometry}

\subsection{Ellipsometry: background}

Ellipsometry is a well established and widely applied tool for the detection and characterization of thin layers on planar interfaces [86]. The technique yields averaged film properties, since in the common reflection geometry there is a light moment transfer $q_{\perp}$ perpendicular to the interface only, while $q_{\|}$is zero. Section 3.2 addresses applications of the ellipsometric measurements scheme to light scattering by rough planar interfaces away from the reflected beam, 
which involves a finite $q_{\|}$and therefore gives access to the in-plane interfacial structure with scattering resolution. A different approach reviewed in Section 3.3 is the ellipsometric polarization analysis of light scattered by spherical colloids. It yields the colloid size and refractive index as well as a characterization of interfacial structures or films on the colloidal particles. The ellipsometric resolution of the film thickness in the nanometer range is far beyond the resolution of conventional light scattering. Ellipsometry can be viewed as an interferometric technique [ $\left.87^{\circ}\right]$, which yields the amplitude ratio tan $(\Psi)$ and the relative phase shift $\Delta$ of two polarization eigen-modes of the system under study. The propagation of the eigen-modes through the optical system is described by the two transfer coefficients $S_{1}$ and $S_{2}$, respectively. These complex coefficients contain amplitude transfer and phase shift of each mode. The ellipsometric coefficient $\rho$ is usually written as

$\rho=\frac{S_{2}}{S_{1}}=\tan (\Psi) \exp [i \Delta]$.

Systems with a partial depolarization of light require a description by the $4 \times 4$ Mueller matrix $[3,4,86]$. In this case, the ellipsometric parameters read $\left[88^{\circ}, 89\right]$

$\tan (2 \Psi)=\frac{2\left|\left\langle S_{1}^{*} S_{2}\right\rangle\right|}{\left\langle\left|S_{1}\right|^{2}\right\rangle-\left\langle\left|S_{2}\right|^{2}\right\rangle}$

$\Delta=\arg \left(\left\langle S_{1}^{*} S_{2}\right\rangle\right)$

Here, $\arg (\cdot)$ denotes the complex argument and $\langle\cdot\rangle$ describes the averaging over system parameters involving a distribution (light wavelength distribution, angle of incidence distribution, particle size distribution). In standard reflection ellipsometry on isotropic interfaces, the eigen-modes are linear $s$ and $p$-polarization perpendicular and parallel to the plane of reflection and $S_{1}$ and $S_{2}$ are the corresponding field amplitude reflectivities $r_{s}$ and $r_{p}$, respectively. For scattering on isotropic samples, the eigen-modes are usually linear polarizations perpendicular to and in plane with the scattering plane, defined by the directions of the incident and the scattered light. The eigen-modes can be different for interface scattering, in case the interface normal is not contained in the scattering plane. $S_{1}$ and $S_{2}$ are the corresponding scattering coefficients. Ellipsometric optics usually consists of a polarizer $(\mathrm{P})$, a quarter wave plate called compensator (C) rotated by $45^{\circ}$ compared to the perpendicularly oriented directions of the two polarization eigen-modes, the sample $(S)$ and an analyzer (A) (PCSA or PSCA geometry, according to the order of components) [86]. In nulling ellipsometry, the intensity is minimized by rotating $\mathrm{P}$ and $\mathrm{A}$, and the resulting nulling values of $\mathrm{P}$ and $\mathrm{A}$ are directly connected to $\Delta$ and $\Psi$. Of high practical importance is twozone averaging, where measurements of two different sets of nulling angles are suitably averaged [86]. Most imperfections and alignment errors of the optical components are eliminated in the two-zone averages resulting in high accuracy ellipsometric data. Ellipsometric measurements for the detection of the complete Mueller matrix are usually based on a procedure developed by Azzam [90*]. Ellipsometric sensitivity on an interface layer is highest either at the Brewster angle or the critical angle of total internal reflection $\varphi_{0}$.

\subsection{Planar interfaces}

Scattering measurements at an interface detect a superposition of scattering contributions from the bulk and from the interface. In principle, bulk contributions are caused by inhomogeneities and concentration or density fluctuations in the bulk, while interface scattering reflects surface roughness or fluctuations in interfacial coverage. Complementary to EWLS is an ellipsometric scattering technique (called scattering ellipsometry (SE) [91 ${ }^{\circ}$ ] or Ellipsometry of Angle-Resolved Scattering (E.A.R.S.) [92]), where a distinction of scattering contributions relies on a polarization analysis of the scattered light. While SE has not yet been applied to colloid and interface science, it has a high potential in this field. Only the main principles and some applications are briefly described here.

The idealizing distinction of an interface as a two-dimensional object and a three-dimensional bulk can be rather arbitrary for specific systems, and in colloid and interface science it is common to address a finite transition zone between two bulk phases as a separate entity called inter-phase. Examples for extended interphases are the polymer brushes and boundary regions with modified dynamics in liquid crystals or colloidal systems, as described in Section 2. Thus, EWLS is a technique to characterize inter-phases rather than interfaces. For SE, on the other hand, roughness is typically considered as a height variation of a step-like transition from one phase to the other. If the height variation is small compared to $\lambda$ with slopes much less than unity and if this is the only scattering source, the scattering can be described by a first order vector perturbation theory (FOVPT) (see e.g. [93]). An important feature of FOVPT which is essential for many applications is a factorization in a polarization term and a term including the surface roughness, described by the mean squared amplitude $|Z(\vec{q})|^{2}$ of the two-dimensional Fourier transform $Z(\vec{q})$ of the interface height $z(x, y)$. Thus, for such rather smooth interfaces the polarization properties of the scattered light depend on the refractive index values, the illumination and detection directions and the polarization of the incident light, however, they are independent of $|Z(\vec{q})|^{2}$. The extinction conditions for the polarization optics therefore do not depend on the roughness. This feature has been used to mask out either bulk or interface scattering, either in scattering measurements $[92,94]$ or in an imaging application [95]. The theoretical description has been generalized to a multi-layer stack with rough interfaces between the layers [96]. Experimentally, FOVPT was confirmed by Germer et al. on a micro-fabricated silicon micro-roughness standard with a pseudo random distribution of circular pits with depth $1 \mathrm{~nm}$ and radius $1.31 \mu \mathrm{m}$ and $1.76 \mu \mathrm{m}$ [97]. In measurements out of the reflection plane, a vanishing of scattering intensity for incident and detected $p$-polarization was observed for certain angles, similar to the vanishing $p$-reflectivity at the Brewster angle. Germer investigated SiO films (thickness $10.3 \mathrm{~nm}$ and $52 \mathrm{~nm}$ ) grown on a microrough Si substrate [91*]. SE yields the correlation of the roughnesses at the two interfaces (substrate/film and film/air), where full correlation with a slightly more rough film/air interface was observed. A further work of Germer demonstrates the distinction of different scattering sources by a polarization analysis of scattering [98*]. Rayleigh defects above, within, or below a surface film and roughnesses at the two film interface have different polarization signatures in scattering measurements out of the reflection plane. A procedure to decompose a Stokes vector into two non-depolarizing sources is described. Finally, the importance of correlation is emphasized: for uncorrelated scattering sources there is an incoherent superposition of intensities and the strongest source dominates.

\subsection{Ellipsometric light scattering}

The size of colloidal particles usually exceeds significantly the molecular length scale of a dispersing liquid. These particles are not solubilized, but dispersed in the liquid medium. Therefore, colloidal interfaces are present in a dispersion. Structure, properties, and dynamics of these interfaces are essential for the colloidal stabilization against particle aggregation. For an investigation of colloidal interfaces, Erbe et al. applied the nulling ellipsometry measurement scheme to a bulk scattering experiment on spherical colloids and established ellipsometric light scattering (ELS) $\left[87^{*}, 89,99,100^{\circ}\right]$. The 
bulk scattering geometry differs from the reflection geometry used in $\mathrm{SE}$ (see Section 3.2). For spherical colloids of about $100 \mathrm{~nm}$ radius, ELS bears close similarities to reflection ellipsometry on planar interfaces [87"*]. A minimum in $\tan (\Psi)$ close to $90^{\circ}$ scattering angle resembles the Brewster angle in reflection ellipsometry, and the sensitivity is highest at this minimum. Location and depth of the minimum are modeled by Mie scattering and contain information on refractive indexes and sizes of core and shell of the particles. A resolution in layer thickness better than $20 \mathrm{~nm}$ was demonstrated by measurements at two $\lambda(532 \mathrm{~nm}, 633 \mathrm{~nm})$ on PS colloids $(R \approx 90 \mathrm{~nm})$ covered by poly( $\mathrm{N}$-isopropylacrylamide) (PNIPAM) shells, where the temperature dependent shell swelling was monitored. In a further project, Erbe et al. applied ELS to characterize the charge distribution around charge stabilized colloids $(R=60 \mathrm{~nm})$ [99]. For bulk salt concentrations above $c^{*}=10^{-4} \mathrm{Mol} / \mathrm{L}$, enhanced local salt concentrations up to $4 \mathrm{Mol} / \mathrm{L}$ in layers of $20-30 \mathrm{~nm}$ thickness at the colloidal interfaces were observed. This local concentration is two orders of magnitude higher than predictions by the Poisson-Boltzmann theory. The observation is discussed as a first order prewetting transition at the colloidal interfaces.

An investigation of monodisperse unilamellar lipid vesicles $(R=45 \mathrm{~nm}$, shell thickness $5 \mathrm{~nm}$ ) by Erbe et al. revealed a high sensitivity of ELS on radially symmetric birefringence [100*]. The detected birefringence gives access to the local order parameter of the lipid chains, where values of 0.65 and 0.32 were observed for 1,2Dipalmitoyl-snglycero-3-phosphocholine (DPPC) vesicles in the gelphase and 1,2-Dioleoyl-sn-glycero-3-phosphocholine (DOPC) vesicles in the liquid crystalline phase, respectively. For DPPC, a lower bound of $29^{\circ}$ for the average chain tilt angle against the surface normal was determined. The ELS sensitivity on local birefringence was further employed by Erbe et al. to determine the local stress in PS colloids $\left(R_{\mathrm{h}}=60 \ldots 97 \mathrm{~nm}\right)$ via the stress-optical coefficient of polymers [89]. The magnitude of the observed birefringence was rationalized as an effect of interface tension during particle synthesis.

In a theoretical contribution, Sigel and Erbe discussed the effect of size polydispersity and beam profile on ELS measurements [ $\left.88^{\circ}\right]$. In analogy to neutron scattering terminology, a distinction between coherent scattering that reflects the averaged particle scattering properties and incoherent scattering as an effect of deviations from the average by individual particles is introduced. The illumination of a scattering volume by a Gaussian beam leads to further incoherent scattering. The ellipsometric parameters $\Delta$ and $\tan \Psi$ are determined solely by the coherent scattering, while a new parameter $\tan \Psi_{I}=\left[\left\langle\left|S_{2}\right|^{2}\right\rangle /\left\langle\left|S_{1}\right|^{2}\right\rangle\right]^{1 / 2}$ contains information on coherent and incoherent contributions. An experimental investigation on colloid mixtures confirms the validity of the theoretical concepts [89]. The distinction of coherent and incoherent contributions is also useful for classic reflection ellipsometry, for a description of rough interfaces and a modeling of the Gaussian beam profile in measurements at the critical angle of total internal reflection [88*].

A comparable technique termed "Mie scattering ellipsometry" (MSE) is used in plasma physics [101,102]. Main focus in MSE applications has been the determination of particle size and size distribution for particles created in plasmas [103-105], e.g. for process control in dust sensitive micro device fabrication. The characterization of particle interfaces is mentioned briefly as a hypothetical application [102] but it is hardly used so far. MSE was used further to monitor the particle content in hot gas entering a gas turbine [106] and for the a characterization of particle sizes in a micro emulsion, where multiple scattering effects were simulated numerically [107]. MSE relies on measurements of the ratio $\tan \left(\Psi_{\mathrm{s}}\right)=E_{s \|} / E_{s \perp}$ of scattered electric field amplitudes $E_{s \|}$ and $E_{S \perp}$ polarized parallel and perpendicular to the scattering plane, respectively. The ratio is measured at three $\lambda$ $(488 \mathrm{~nm}, 514 \mathrm{~nm}, 633 \mathrm{~nm})$ and a single scattering angle (typically $90^{\circ}$ ) by a rotating compensator technique for a series of polarization angles $P$ of the incident linear light polarization against the scattering plane.
Some technical background is found in the PhD thesis of Gebauer [108]. The connection to ELS reads $\tan \left(\Psi_{s}\right)=\tan (\Psi) / \tan (P)$.

Scattering measurements on the complete Mueller matrix have been performed by Volten et al. on prolate birefringent rutile particles (size roughly $220 \mathrm{~nm}$ ) [109] and by Kaplan et al. [110] for spherical PS colloids $(2 R=400 \ldots 3000 \mathrm{~nm})$. While the detailed scattering properties are of interest by themselves, there is only limited know-how on the inverse problem to learn something about the particles and their interfaces from such scattering measurements with maximum polarization information.

\section{Conclusions and outlook}

This synopsis of interface light scattering investigations elucidates common and distinct features of different soft matter classes situated close to interfaces. While the wall drag effect has been the focus in investigations on colloidal particles at interfaces, it is not at all considered for polymer brushes, which are described on a short scale given by the correlation length of a semidilute polymer solution. For liquid crystals and their orientational wetting, in turn, surface-bound fluctuation modes and special scattering properties in a steady refractive index profile were under study. This spread of different description and effects indicates, that the usual fruitful exchange in soft matter science between the fields of polymers, colloids, and liquid crystals is only partly established for the interfacial behavior. Improved exchange could lead to a further fertilization and a better understanding of interfacial soft matter behavior, and the overview compiled in this review might serve as a road map.

EWLS extends the power of bulk light scattering to investigations at planar interfaces. The technique is significantly more demanding than bulk scattering measurements. From the experimental point of view, the quality of the interface is essential. A full independent access to penetration depth and scattering vector components parallel and perpendicular to the interface requires a threedimensional scattering instrument, the alignment of which is more delicate than for bulk scattering setups. The theoretical description for data interpretation is more involved than the well-behaved Fourier transform which is the basis for bulk scattering analysis. Despite these difficulties, EWLS has grown to a mature technique, as indicated by the regular high quality publications. For the investigation of interfaces of spherical colloids, ELS is a young yet powerful technique. Its high resolution and the sensitivity to local birefringence and thus local stress open a new and promising experimental access to colloidal interfaces. The application of scattering ellipsometry to planar soft matter interfaces appears as a treasure not jet uncovered.

Recently, Granick et al. reviewed on-going and recommended research directions of macromolecules at interfaces [111]. The review is a product of a study panel commissioned by the U.S. Department of Energy Council on Materials. Beside synthesis issues, the understanding of structure and dynamics of synthetic or naturally occurring macromolecules at interfaces is recognized as an area of high interest. Clearly, the techniques for interface sensitive light scattering summarized here are predestined to give a significant contribution in this area. Further, Granick et al. suggest neutron scattering measurements with EW illumination to study interfacial phenomena on a short length scale. The know-how collected with EWLS can serve as a solid basis for an application of the principles to such an ambitioned target.

\section{Acknowledgements}

The author thanks Benoit Loppinet for fruitful discussions and Gerd Gebauer for clarifying remarks concerning MSE. Financial support of the Adolphe Merkle Foundation is gratefully acknowledged. 


\section{References}

[1] Berne BJ, Pecora R. Dynamic light scattering. Mineola: Dover Publications; 2000.

[2] Brown W, editor. Dynamic light scattering. Clarendon Oxford; 1993.

[3] Kerker M. The scattering of light and other electromagnetic radiation. San Diego: Academic Press; 1969.

[4] Bohren CF, Huffman DR. Absorption and scattering of light by small particles. New York: Wiley; 1998.

[5] Scheffold F, Cerbino R. New trends in light scattering. Curr Opin Colloid Interface Sci 2007;12:50-7.

[6] Prieve DC, Frej NA. Total internal-reflection microscopy - a quantitative tool for the measurement of colloidal forces. Langmuir 1990;6:396-403.

[7] Bike SG. Measuring colloidal forces using evanescent wave scattering. Curr Opin Colloid Interface Sci 2000;5:144-50.

[8] Vollmer F, Arnold S. Whispering-gallery-mode biosensing: label-free detection down to single molecules. Nat Methods 2008;5:591-6.

[9] Armani AM, Kulkarni RP, Fraser SE, Flagan RC, Vahala KJ. Label-free, singlemolecule detection with optical microcavities. Science 2007;317:783-7.

[10] Keng D, McAnanama SR, Teraoka I, Arnold S. Resonance fluctuations of a whispering gallery mode biosensor by particles undergoing Brownian motion. Appl Phys Lett 2007;91:103,902.

[11] Langevin D, editor. Light scattering by liquid surfaces and complementary techniques. New York: Marcel Dekker; 1992.

[12] Earnshaw JC. Light scattering as a probe of liquid surfaces and interfaces. Adv Colloid Interface Sci 1996;68:1-29.

[13] Cicuta P, Hopkinson I. Recent developments of surface light scattering as a tool for optical-rheology of polymer monolayers. Colloids Surf, A 2004;233:97-107.

[14] Lan KH, Ostrowsky N, Sornette D. Brownian dynamics close to a wall studied by photon correlation spectroscopy from an evanescent wave. Phys Rev Lett 1986;57:17-20. Pioneering work where EWDLS was used for the first time as a probe for interfacial sample dynamics. The mirror effect on colloidal diffusion and the effect of the inhomogeneous illumination are discussed, the wall drag effect for colloidal diffusion on EWDLS measurements is outlined.

[15] Marcus AH, Lin BH, Rice SA. Self-diffusion in dilute quasi-two-dimensional hard sphere suspensions: evanescent wave light scattering and video microscopy studies. Phys Rev, E 1996;53:1765-76. A summary of the theoretical expectation for two dimensional diffusion complemented by experiments.

[16] Born M, Wolf E. Principles of optics. 7th ed. Cambridge University Press; 1999.

[17] Stocco A, Tauer K, Pispas S, Sigel R. Dynamics at the air-water interface revealed by evanescent wave light scattering. Eur Phys J, E 2009;29:95-105. The work outlines a mechanism of enhanced scattering when the angle of incidence equals the critical angle of total reflection.

[18] Harrick NJ. Internal reflection spectroscopy. New York: Wiley; 1976.

[19] Holmqvist P, Dhont JKG, Lang PR. Anisotropy of Brownian motion caused only by hydrodynamic interaction with a wall. Phys Rev, E 2006;74:021402.

[20] Potenza MAC, Brogioli D, Giglio M. Total internal reflection scattering. Appl Phys Lett 2004;85:2730-2.

[21] Sigel R, Strobl G. Static and dynamic light scattering from the nematic wetting layer in an isotropic liquid crystal. Prog Colloid \& Polym Sci 1997;104:187-90. The fluctuation dynamics in a nematic liquid crystalline wetting layer were detected experimentally. EWDLS was shown to work even for layers as thin as a few nanometers.

[22] Sigel R, Strobl G. Light scattering by fluctuations within a nematic wetting layer in a isotropic phase of a liquid crystal. J Chem Phys 2000;112:1029-39.

[23] Plum MA, Steffen W, Fytas G, Knoll W, Menges B. Probing dynamics at interfaces: resonance enhanced dynamic light scattering. Opt Express 2009;17:10,364-71.

[24] Rothenhausler B, Knoll W. Plasmon surface polariton fields versus tir evanescent waves for scattering experiments at surfaces. Opt Commun 1987;63:301-4.

[25] Ostrowsky N, Garnier N. Brownian dynamics close to a wall, measured by quasielastic light-scattering from an evanescent wave. Prog Colloid \& Interf Sci 1991;84:371-6.

[26] Garnier N, Ostrowsky N. Brownian dynamics in a confined geometry experiments and numerical simulations. J Phys, II 1991;1:1221-32.

[27] Israelachvili J. Intermolecular and surface forces. Amsterdam: Academic Press; 1991.

[28] Gaigalas AK, Hubbard JB, Plant AL, Reipa V. Application of evanescent-wave scattering to the study of the motion of liposomes near a solid-surface. J Colloid Interface Sci 1995; 175:181-9.

[29] Hosoda M, Sakai K, Takagi K. Measurement of anisotropic Brownian motion near an interface by evanescent light-scattering spectroscopy. Phys Rev, E 1998;58: 6275-80.

[30] Matsuoka H, Morikawa H, Tanimoto S, Kubota A, Naito Y, Yamaoka H. Evaluation of the dynamic properties of polymer latex particles interacting with quartz interface by evanescent wave dynamic light scattering. Colloid Polym Sci 1998;276:349-55.

[31] Matsuoka $\mathrm{H}$. Evanescent wave light scattering - a fusion of the evanescent wave and light scattering techniques to the study of colloids and polymers near the interface. Macromol Rapid Commun 2001;22:51-67.

[32] Feitosa MIM, Mesquita ON. Wall-drag effect on diffusion of colloidal particles near surfaces - a photon-correlation study. Phys Rev, A 1991;44:6677-85.

[3.3] Holmqvist P, Dhont JKG, Lang PR. Colloidal dynamics near a wall studied by evanescent wave light scattering: experimental and theoretical improvements

\section{- Of special interest}

- Of outstanding interest. and methodological limitations. J Chem Phys 2007;126:044707. With a threedimensional scattering instrument, the scattering vectors $q_{\|}$and $q_{\perp}$ parallel and perpendicular to the interface in an evanescent wave geometry were varied independently to distinguish colloidal diffusion parallel and perpendicular to the interface. The wall drag effects in the two directions were separated. This work provides a more comprehensive description of the experiment and the theoretical calculation of the initial slope of the correlation function for an evanescent wave geometry than reference [19], where the authors applied and briefly described the approach for the first time.

[34] Holmqvist P, Kleshchanok D, Lang PR. Unexpected slow near wall dynamics of spherical colloids in a suspension of rods. Langmuir 2007;23:12,010-5.

[35] Holmqvist P, Kleshchanok D, Lang PR. Interaction potential and near wall dynamics of spherical colloids in suspensions of rod-like fd-virus. Eur Phys J, E 2008;26:177-82

[36] Albery WJ, Kneebone GR, Foulds AW. Kinetics of colloidal deposition studied by a wall-jet cell. J Colloid Interface Sci 1985;108:193-8.

[37] Albery WJ, Fredlein RA, Kneebone GR, O'Shea GJ, Smith AL. The kinetics of colloidal deposition under conditions of controlled potential. Colloids Surf 1990;44:337-56

[38] Schumacher G, van de Ven TGM. Evanescent wave scattering studies on latexglass interactions. Langmuir 1991:7:2028-33.

[39] Xia ZM, van de Ven TGM. Adhesion kinetics of phosphatidylcholine liposomes by evanescent wave light-scattering. Langmuir 1992;8:2938-46.

[40] Polverari M, van de Ven TGM. Electrostatic and steric interactions in particle deposition studied by evanescent-wave light-scattering. J Colloid Interface Sci $1995 ; 173: 343-53$. This work on colloids is a suitable entry point to investigations on interfacial adsorption based on a combination of EWLS and stagnation point flow.

[41] Polverari M, van de Ven TGM. Effect of flow on the distribution of colloidal particles near surfaces studied by evanescent-wave light-scattering. Langmuir 1995;11:1870-6.

[42] Pouligny B, Aastuen DJW, Clark NA. Total-internal-reflection study of a colloidalcrystal container-wall interface. Phys Rev, A 1991;44:6616-25.

[43] Schmidt M, Krieger S, Johannsmann D. Film formation of latex dispersions observed with evanescent dynamic light scattering. Prog Colloid \& Polym Sci 1997; $104: 191-3$

[44] Voudouris P, Loppinet B, Petekidis G. Particle dynamics within a wetting layer in a colloid-polymer mixture. Phys Rev, E 2008;77:051402.

[45] Bonn D, Wegdam GH. Viscoelastic interfacial modes in a wetting layer. Phys Rev, E 1993;48:350-6.

[46] Michailidou VN, Petekidis G, Swan JW, Brady JF. Dynamics of concentrated hardsphere colloids near a wall. Phys Rev Lett 2009;102:068302. A screening of the wall drag effect for enhanced concentration is demonstrated. The supplementary information contains the calculation of the initial slope of the correlation function in evanescent wave illumination for enhanced concentration.

[47] Chew H, Wang DS, Kerker M. Elastic scattering of evanescent electromagnetic waves. Appl Opt 1979;18:2679-87.

[48] Liu C, Kaiser T, Lange S, Schweiger G. Structural resonances in a dielectric sphere illuminated by an evanescent wave. Opt Commun 1995;117:521-31.

[49] Quinten M, Pack A, Wannemacher R. Scattering and extinction of evanescent waves by small particles. Appl Phys, B 1999;68:87-92.

[50] Kawata S, Sugiura T. Movement of micrometer-sized particles in the evanescent field of a laser beam. Opt Lett 1992;17:772-4. Experimental observation of colloida movement at the interface induced by the light pressure in an evanescent wave geometry.

[51] Almaas E, Brevik I. Radiation forces on a micrometer-sized sphere in an evanescent field. J Opt Soc Am, B 1995;12:2429-38.

[52] Chang S, Jo JH, Lee SS. Theoretical calculations of optical force exerted on a dielectric sphere in the evanescent field generated with a totally-reflected focused Gaussian beam. Opt Commun 1994;108:133-43.

[53] Chang S, Kim JT, Jo JH, Lee SS. Optical force on a sphere caused by the evanescent field of a Gaussian beam; effects of multiple scattering. Opt Commun 1997: 252-61.

[54] Zvyagin AV, Goto K. Mie scattering of evanescent waves by a dielectric sphere: comparison of multipole expansion and group-theory methods. J Opt Soc Am 1998; 15 Am A 3003-8.

[55] Pendleton JD. Mie scattering into solid angles. J Opt Soc Am 1982;71:1029-33.

[56] Liu C, Weigel T, Schweiger G. Structural resonances in a dielectric sphere on a dielectric surface illuminated by an evanescent wave. Opt Commun 2000;185: 249-61.

[57] Doicu A, Wriedt T, Eremin YA. Light scattering by systems of particles. Berlin: Springer; 2006.

[58] Ganic D, Gan X, Gu M. Three-dimensional evanescent wave scattering by dielectric particles. Optik 2002;113:135-41.

[59] Wu HJ, Shah S, Beckham R, Meissner KE, Bevan MA. Resonant effects in evanescent wave scattering of polydisperse colloids. Langmuir 2008;24: 13,790-5.

[60] Eichmann SL, Anekal SG, Bevan MA. Electrostatically confined nanoparticle interactions and dynamics. Langmuir 2008;24:714-21.

[61] Selser JC, Rothschild KJ, Swalen JD, Rondelez F. Study of multilamellar films of photoreceptor membrane by photon-correlation spectroscopy combined with integrated optics. Phys Rev Lett 1982;48:1690-3. First work in total on EWDLS.

[62] Lobry L, Ostrowsky N. Diffusion of Brownian particles trapped between two walls: theory and dynamic-light-scattering measurements. Phys Rev B 1996;53:12,050-6. The drag of two walls confining a colloidal sphere to a 
quasi two-dimensional geometry is calculated in an elegant way. Experimental results fit well to the prediction.

[63] Benesch T, Yiacoumi S, Tsouris C. Brownian motion in confinement. Phys Rev, E 2003;68:021401.

[64] Gao J, Rice SA. Light scattering with incident evanescent waves: a method for studying the properties of adsorbed polymers. J Chem Phys 1989;90:3469-78.

[65] Gao J, Rice SA. Light scattering with evanescent waves: intermolecular interference and the structure factor for an ideal flexible chain at an interacting interface. J Chem Phys 1990;93:2785-800.

[66] Rosa E, Dahler JS. The scattering of evanescent light waves from oriented, tethered rod polymers. J Chem Phys 1992;97:1-3.

[67] Fytas G, Anastasiadis SH, Seghrouchni R, Vlassopoulos D, Li JB, Factor BJ, et al. Probing collective motions of terminally anchored polymers. Science 1996;274:2041-4. First measurements on an adsorbed brush. The observed rather slow dynamics was identified in a later theoretical work [76] as an anchorsliding mode.

[68] Loppinet B, Petekidis G, Fytas G, Rulkens R, Wegner G. Dynamics of adsorbed hairy-rod polymer solutions. Langmuir 1998;14:4958-60.

[69] Yakubov GE, Loppinet B, Zhang H, Rühe J, Sigel R, Fytas G. Collective dynamics of an end-grafted polymer brush in solvents of varying quality. Phys Rev Lett 2004:92:115,501. First investigations of brush dynamics with a covalently anchored brush. The fast cooperative dynamics is complemented by a slow relaxation process for a theta solvent.

[70] Michailidou VN, Loppinet B, Prucker O, Rühe J, Fytas G. Cooperative diffusion of end-grafted polymer brushes in good solvents. Macromolecules 2005;38: 8960-2.

[71] Michailidou VN, Loppinet B, Vo DC, Prucker O, Rühe J, Fytas G. Dynamics of endgrafted polystyrene brushes in theta solvents. J Polym Sci, B 2006;44:3590-7.

[72] Filippidi E, Michailidou V, Loppinet B, Ruhe J, Fytas G. Brownian diffusion close to a polymer brush. Langmuir 2007;23:5139-42. The work provides a comparison of the diffusion of hard and soft particles close to an interface covered with a polymer brush.

[73] Michailidou VN, Loppinet B, Vo CD, Ruhe J, Tauer K, Fytas G. Observation of slow down of polystyrene nanogels diffusivities in contact with swollen polystyrene brushes. Eur Phys J, E 2008;26:35-41.

[74] Likhtman AE, Anastasiadis SH, Semenov AN. Theory of surface deformations of polymer brushes in solution. Macromolecules 1999;32:3474-80.

[75] Anastasiadis SH. Collective dynamics of terminally anchored polymer chains. Macromol Symp 1999;139:31-7.

[76] Semenov AN, Anastasiadis SH. Collective dynamics of polymer brushes. Macromolecules 2000;33:613-23.

[77] Lin BH, Rice SA, Weitz DA. Static and dynamic evanescent-wave light-scatteringstudies of diblock copolymers adsorbed at the air-water-interface. J Chem Phys 1993;99:8308-24.

[78] Roovers J. Dillute-solution properties of ring polystyrenes. J Polym Sci, Polym Phys Ed 1985;23:1117-23.

[79] Copic M, Park CS, Clark NA. Theory of evanescent light-wave scattering at the solid-nematic interface. Mol Cryst Liquid Cryst 1992;223:111-8.

[80] Copic M, Clark NA. Theory of orientational modes at a nematic solid interface when do surface-modes appear. Liquid Cryst 1994;17:149-55.

[81] Park CS, Copic M, Mahmood R, Clark NA. Dynamic behavior at a nematic liquidcrystal rubbed nylon interface using evanescent-wave photon-correlation spectroscopy. Liquid Cryst 1994;16:135-42.

[82] Lin BH, Rice SA. Evanescent wave light-scattering study of a diblock copolymer adsorbed at the air-water-interface. J Chem Phys 1993;98:6561-3.

[83] Lin BH, Rice SA Weitz DA. Experimental-evidence for the divergence of a transport coefficient in a quasi-2-dimensional fluid. Phys Rev, E 1995;51:423-9. The contribution describes the diffusion and its slowing down at low $q_{\|}$for insoluble block copolymers forming "furry discs" at a water-air interface.

[84] Stocco A, Mokhtari T, Haseloff G, Erbe A, Sigel R. Evanescent wave dynamic light scattering at an oil-water interface: diffusion of interface adsorbed colloids. Phys Rev E, submitted.

[85] Pieranski P. Two-dimensional interfacial colloidal crystals. Phys Rev Lett 1980;45:569-72.

[86] Azzam RMA, Bazhara NM. Ellipsometry and polarized light. Amsterdam: Elsevier; 1977.

[87] Erbe A, Tauer K, Sigel R. Ellipsometric light scattering for the characterization of thin layers on dispersed colloidal particles. Phys Rev, E 2006;73:031406. Introduction of ELS and comparison of the Brewster angle in ELS and reflection ellipsometry. The ELS resolution was demonstrated with a layer of a thermoresponsible polymer.

[88] Sigel R, Erbe A. Effects of sample polydispersity and beam profile on ellipsometric light scattering. Appl Opt 2008;47:2161-70. Theory for ELS and ellipsometry on partially depolarizing samples.

[89] Erbe A, Tauer K, Sigel R. Separation of coherent and incoherent scattering contributions in ellipsometric light scattering experiments on latex mixtures. Langmuir 2009;25:2703-10.

[90] Azzam RMA. Photopolarimetric measurement of Mueller matrix by Fourieranalysis of a single detected signal. Opt Lett 1978;2:148-50. An early and important procedure to measure the full Mueller matrix of an optical system is described.

[91] Germer TA. Measurement of roughness of two interfaces of a dielectric film by scattering ellipsometry. Phys Rev Lett 2000;85:249-52. The roughness correlation at the two interfaces of a film was detected by scattering ellipsometry.

[92] Gilbert O, Deumié C, Amra C. Angle-resolved ellipsometry of scattering patterns from arbitrary surfaces and bulks. Opt Express 2005;13:2403-18.

[93] Amra C, Grezes-Besset C, Bruel L. Comparison of surface and bulk scattering in optical multilayers. Appl Opt 1993;32:5492-503.

[94] Amra C, Deumié C, Gilbert O. Elimination of polarized light scattered by surface roughness or bulk heterogeneity. Opt Express 2005:13:10,854-64.

[95] Georges G, Deumié C, Amra C. Selective probing and imaging in random media based on the elimination of polarized scattering. Opt Express 2007;15:9804-16.

[96] Amra C. First-order vector theory of bulk scattering in optical multilayers. J Opt Soc Am A 1993:10:365-74

[97] Germer TA, Asmail CC, Scheer BW. Polarization of out-of-plane scattering from microrough silicon. Opt Lett 1997;22:1284-6.

[98] Germer TA. Polarized light scattering by microroughness and small defects in dielectric layers. J Opt Soc Am A 2001;18:1279-88. Summary of the theory for different scattering sources connected to a film on a planar interface and experimental demonstration that the sources can be distinguished by scattering ellipsometry.

[99] Erbe A, Tauer K, Sigel R. Ion distribution around electrostatically stabilized polystyrene latex particles studied by ellipsometric light scattering. Langmuir 2007;23:452-9.

[100] Erbe A, Sigel R. Tilt angle of lipid acyl chains in unilamellar vesicles determined by ellipsometric light scattering. Eur Phys J, E 2007;22:303-9. The effect of a radial symmetric birefringence on ELS was demonstrated experimentally. Details of the structure of a thin vesicle membrane are extracted from the data.

[101] Hayashi Y, Tachibana K. Mie-scattering ellipsometry for analysis of particle behaviors in processing plasmas Japanese J Appl Phys 2 Lett 1994;3B:L476-8.

[102] Gebauer G, Winter J. In situ nanoparticle diagnostics by multi-wavelength Rayleigh-Mie scattering ellipsometry. New J Phys 2003;5(38):1-17.

[103] Swinkels GHPM, Stoffels E, Stoffels WW, Simons N, Kroesen GMW, de Hoog FJ. Treatment of dust particles in an RF plasma monitored by Mie scattering rotating compensator ellipsometry. Pure Appl Chem 1998;70:1151-5.

[104] Hong SH, Winter J. Size dependence of optical properties and internal structure of plasma grown carbonaceous nanoparticles studied by in situ Rayleigh-Mie scattering ellipsometry. J Appl Phys 2006;100:064303.

[105] Weiss R, Hong SH, Ränsch J, Winter J. Rayleigh-Mie scattering ellipsometry as an in situ diagnostic for the production of "smart nanoparticles". Phys Status Solidi, A 2008;205:802-5.

[106] Gebauer G, Mian I, Förster MEC, Bartmann R, Telöken, Naumann F, et al. Onlinein-situ-Partikelmesstechnik mittels Mie-Ellipsometrie an der Druckkohlenstaubfeuerungsanalge in Dorsten Chemie Ingeneur Technik, vol. 77; 2005. p. 1601-8.

[107] Gebauer G. Online-in-situ-Mikroemulsionsmesstechnik. Chemie Ingeneur Technik, vol. 78; 2006. p. 1544-9.

[108] Gebauer G, In situ Nano-Partikel Diagnostik in Plasma durch Mie-Ellipsometrie. PhD thesis, University Bochum, Germany, 2001.

[109] Volten H, Jalava JP, Lumme K, de Haan JF, Vassen W, Hovenier JW. Laboratory measurements and T-matrix calculations of the scattering matrix of rutile particles in water. Appl Opt 1999;38:5232-40.

[110] Kaplan B, Compain E, Drevillon B. Phase-modulated Mueller ellipsometry characterization of scattering by latex sphere suspensions. Appl Opt 2000;39: 629-36.

[111] Granick S, Kumar SK, Amis EJ, Antonietti M, Balazs AC, Chakraborty AK, et al. Macromolecules at surfaces: research challenges and opportunities from tribology to biology. J Polym Sci 2003;41:2755-93. 